\title{
Mu-opioid Self-Administration vs Passive Administration in Heroin Abusers Produces Differential EEG Activation
}

\author{
Mark K Greenwald*,' and Timothy A Roehrs ${ }^{2}$ \\ 'Substance Abuse Research Division, Department of Psychiatry and Behavioral Neurosciences, Wayne State University School of Medicine, \\ Detroit, MI, USA; ${ }^{2}$ Sleep Disorders and Research Center, Henry Ford Hospital, Detroit, MI, USA
}

\begin{abstract}
Psychoactive drug self-administration (SA) produces different neurobiological effects than passive administration (PA) in non-human animals; however, such consequences have never been examined in human drug abusers. The present study compared electroencephalographic (EEG) activation produced by intravenous PA and SA of the mu-opioid fentanyl in eight heroin-dependent, methadone-stabilized male participants. In phase I, participants received cumulative PA of fentanyl (up to $1.5 \mathrm{mg} / 70 \mathrm{~kg}$; session I), then bolus PA of placebo and fentanyl $1.5 \mathrm{mg} / 70 \mathrm{~kg}$ (session 2). High-dose fentanyl significantly increased the amplitude of slow-frequency (delta- and theta-band) EEG activity. In phase 2, bolus fentanyl $1.5 \mathrm{mg} / 70 \mathrm{~kg}$ was available for SA, requiring the participant to complete 1500 responses, in each of two sessions after saline or naloxone pretreatment. Delta EEG peak amplitude increases were greater following fentanyl SA than fentanyl PA, primarily over the central midline region, and were attenuated by naloxone pretreatment. The EEG increase and its attenuation by naloxone agree with preclinical evidence and suggest that SA-related EEG responses were mediated by opioid receptors.

Neuropsychopharmacology (2005) 30, 2 I2-22I, advance online publication, 27 October 2004; doi: I 0.1 038/sj.npp. I 300596
\end{abstract}

Keywords: opioid dependence; self-administration; EEG; fentanyl; naloxone; reinforcement

\section{INTRODUCTION}

Drug dependence is a persistent behavioral disorder of cyclical drug self-administration (SA) despite long-term aversive consequences, and a neurobiological disorder that involves central nervous system adaptations to chronic drug exposure. Drug SA behaviors are maintained by direct pharmacological effects and by stimuli that predict drug availability. Recent evidence suggests that drug SA by laboratory animals engages brain processes independent of processes related to the drug's pharmacological effects from passive administration (PA) (Jacobs et al, 2003). Nonhuman studies have examined neurobiological differences between drug SA and PA using a design that involves triads of rats. One rat is trained to self-administer drug; the second animal receives the same passively administered drug doses in the same temporal pattern and the third animal receives placebo passively in the same temporal pattern. This yokedcontrol method separates the effects of behavioral drug

\footnotetext{
*Correspondence: Dr MK Greenwald, Substance Abuse Research Division, Department of Psychiatry and Behavioral Neurosciences, Wayne State University, 2761 E Jefferson Ave, Detroit, MI 48207, USA, Tel: 001313993 3965, Fax: 001313993 1372,

E-mail: mgreen@med.wayne.edu

Received 7 April 2004; revised 16 August 2004; accepted 15 September 2004

Online publication: 23 September 2004 at http://www.acnp.org/citations/ Npp092304040 16 I/default.pdf
}

responding (first rat) from direct pharmacological effects (second rat), relative to the absence of behavioral responding and drug effect (third rat). These studies have investigated the neurobiological effects of opioids (heroin and morphine), psychostimulants (cocaine and amphetamines), and nicotine. After it was reported that cocaine PA produced greater lethality than cocaine SA (Dworkin et al, 1995), indicating a profound consequence of this behavioral difference, preclinical research on this topic notably increased.

In opioid studies, self-administering animals exhibit higher rates of drug-lever responding than yoked animals (Amit et al, 1976; Welzl et al, 1989). EEG activity in animals is greater preceding morphine SA than PA (Grasing and Szeto, 1993). Animals that self-administer $v s$ receive opioids passively show differential electrophysiological activity of cells in the ventral tegmental area (Kiyatkin and Rebec, 2001) and prefrontal cortex (Lee et al, 1999), as well as differences in nucleus accumbens dopamine release (Hemby et al, 1995) and neurotransmitter turnover rates (Smith et al, 1980, 1982, 1984a). Chronic morphine SA relative to PA alters acetylcholine and benzodiazepine receptor density (Smith et al, 1984b), and the expression of various proteins (Self et al, 1995) and gene transcripts in the nucleus accumbens shell (Jacobs et al, 2002) but not nucleus accumbens core (Jacobs et al, 2004) relative to chronic PA.

Neurobiological differences between drug SA and PA have never been investigated in humans. The investigation of this 
issue is timely because epidemiological data indicate that the abuse of heroin and prescription opioids and their resulting morbidities continue to increase (National Institute on Drug Abuse, 2002). The present study sought to determine whether intravenous SA and PA of the $m u$-opioid (ie heroin-like) drug fentanyl produce different neurophysiological effects in heroin-dependent, methadone-maintained volunteers. Specifically, the study examined whether fentanyl's EEG effects differ with SA vs PA and whether fentanyl produces frequency- and scalp site-specific EEG changes. EEG spectral activity was measured, initially during PA of placebo and fentanyl, followed by sessions in which the same participants responded to self-administer fentanyl. This within-subject design differs from the yokedcontrol between-subjects triad design in non-human studies, but these three experimental conditions (drug SA, drug PA, and placebo PA) are comparable. In addition, the present study examined whether pretreatment with the opioid antagonist naloxone would attenuate EEG activation by fentanyl SA, and to determine whether this effect is mediated by opioid receptors. A physiological reference measure, pupil diameter, was also included to assess whether the effects of opioid SA relative to PA were specific to EEG activity.

Morphine-like drugs can increase EEG power in the delta $(0.35-3.5 \mathrm{~Hz})$ and theta frequency range $(3.5-8 \mathrm{~Hz})$ or decrease power in the alpha-band range $(8-13 \mathrm{~Hz})$ in awake, opioid-abusing individuals (Fink et al, 1971; Phillips et al, 1994; Volavka et al, 1970). Experimenter-administered fentanyl and other short-acting $m u$-opioid analgesics primarily increase delta-band power in surgical patients (Bovill et al, 1983; Scott et al, 1991; Sebel et al, 1981; Wauquier et al, 1984). Fentanyl is extremely lipophilic and, when given intravenously, rapidly crosses the blood-brain barrier to produce EEG effects almost immediately (Scott et al, 1991; Sebel et al, 1981). Although subjective effects and some physiological effects of short-acting $m u$-opioids have been studied in opioid abusers (Greenwald et al, 1996; Baylon et al, 2000), EEG effects of these drugs have not been studied in opioid abusers. It is preferable to evaluate these EEG effects in opioid abusers because, relative to opioidinexperienced individuals, they more consistently respond with a profile of psychopharmacological effects that are related to the abuse liability (ie illicit SA) of these compounds.

\section{METHODS}

\section{Participant Selection and Characteristics}

The Wayne State University Investigational Review Board approved this protocol. This experiment was carried out in accordance with the Declaration of Helsinki as adopted and promulgated by the National Institutes of Health. Heroindependent volunteers were recruited from the Detroit area by advertisements and word of mouth. Volunteers provided a medical history, blood and urine samples for routine laboratory testing, and they received an electrocardiogram, tuberculin screening, and a physical examination. Those selected were not seeking drug abuse treatment, had no chronic diseases, were not taking prescribed medications, and were willing to participate in a short-term research study while receiving methadone. An experienced clinician interviewed all volunteers using the Structured Clinical Interview for DSM-IV (SCID; First et al, 1996). Volunteers were excluded if they reported a current Axis I psychiatric disorder except opioid and nicotine dependence, or were cognitively impaired (estimated IQ $<80$ ) on the Shipley (1967) Institute of Living Scale. During screening, volunteers were required to provide a urine sample that tested positive for opioids or methadone $(>300 \mathrm{ng} / \mathrm{ml})$, and negative for cocaine metabolites, benzodiazepines, and barbiturates $(<300 \mathrm{ng} / \mathrm{ml})$. Volunteers were also required to provide an alcohol-free breath sample. After the procedures were fully explained, all participants provided written informed consent and were paid a flat-rate money amount for completing each session. Volunteers were informed that their methadone dose would be kept constant during their research participation, then tapered over about 4 weeks after completing their participation.

A total of 17 people (16 male, one female) were screened, and three were initially excluded for medical reasons. In all, 14 males enrolled; of these, three dropped out after receiving a few methadone doses and three others began testing but were excluded, either for unsystematic questionnaire responses in session 1, fentanyl-related respiratory depression in session 1, or naloxone-related discomfort in session 3, respectively. Eight (three Black, three white, two Hispanic) males completed all sessions. These eight participants were 39.9 \pm 4.7 (mean \pm SD) years old and had completed $11.5 \pm 1.5$ years of education. Participants reported using heroin daily for $14.9 \pm 9.7$ years and currently used 5.6 $\pm 2.8 \$ 10$-dollar bags of heroin per day (ie spent an average of $\$ 392 /$ week). Five inhaled, two injected, and one inhaled and injected heroin. One participant reported infrequent cocaine use, which did not interfere with testing. Three participants reported sporadic marijuana use (2-7 days in the past month) and one participant reported daily marijuana use. Participants smoked 14.4 \pm 9.3 tobacco cigarettes/day. The first volunteer was maintained on $70 \mathrm{mg} /$ day methadone and the remaining seven volunteers were maintained on $60 \mathrm{mg} / \mathrm{day}$ methadone. Participants weighed $78.9 \pm 15.3 \mathrm{~kg}$.

\section{Drug Administration}

All drugs were administered under double-blind conditions. Methadone hydrochloride $(10 \mathrm{mg} / \mathrm{ml}$; Roxane Pharmaceuticals) was diluted with distilled water to a constant $50-\mathrm{ml}$ volume for all volunteers. Fentanyl hydrochloride $(0.05 \mathrm{mg} /$ ml; ESI/Lederlie/Baxter, Deerfield, IL) and naloxone hydrochloride $(0.4 \mathrm{mg} / \mathrm{ml}$; Endo, Chaddis Ford, PA) were diluted with physiological saline to deliver a constant infusion volume for all drug administrations to each participant. Fentanyl, naloxone, and saline were administered i.v. using an infusion pump. Drug infusion duration was about 2 min and constant within participants, but infusion duration varied slightly across participants because the volume depended on the participant's weight.

\section{Procedure}

General procedures. This EEG study was part of a larger protocol that examined issues of drug craving and seeking 
behavior and vital signs; those data are reported elsewhere (Greenwald, 2004, in press) and do not overlap with the present report.

Participants were maintained on oral methadone for at least 2 weeks prior to the first experimental session. All test sessions started 20-24 h since the previous day's methadone dose (ie at trough level) to minimize cross tolerance to fentanyl's effects while avoiding clinically significant levels of withdrawal signs/symptoms. Prior to each test session, volunteers arrived at the laboratory (the ambulatory side of a hospital-based outpatient research unit) at about 0730 hours. Participants were led to a private room with two-way audio contact and a video camera to monitor their behavior. A bed, drug infusion pump, and vital signs monitoring equipment were also located in the test room.

Before each session, urine and breath samples were collected. All breath samples had to be negative for alcohol. On test session days, urine specimens were required to test negative for cocaine metabolites, benzodiazepines, and barbiturates, whereas opioid-positive urine specimens were allowed but subjected to semiquantitative analysis (see Greenwald, 2002). (About 77\% of all urine samples tested opioid positive prior to experimental sessions. Since fentanyl is rapidly eliminated from the urine, most opioid-positive urine specimens were undoubtedly due to outpatient heroin use. However, no participant arrived at the laboratory for a test session with clinically or statistically significant opioid agonist effects, as confirmed by observation and systematic questionnaire evaluation of session baseline symptoms and vital signs (see Greenwald, 2004, in press)). Second, the nurse inserted the i.v. catheter and started a saline drip. Third, EEG sensors were attached. Fourth, participants were permitted to smoke one cigarette to minimize the potential influence of acute nicotine agonist or abstinence effects during the test sessions. Nicotine and caffeine intake was restricted starting $30 \mathrm{~min}$ before baseline testing until the end of each session. No one reported nicotine or caffeine withdrawal discomfort from these restrictions. Finally, participants were given a snack before the session.

Phase 1 evaluated EEG and pupillary effects of passively administered fentanyl doses (saline and cumulative 0.25 , 0.75 , and $1.5 \mathrm{mg} / 70 \mathrm{~kg}$ in session 1 ; saline and bolus fentanyl $1.5 \mathrm{mg} / 70 \mathrm{~kg}$ in session 2). Session 1 was designed to evaluate the EEG effects of a wide range of ascending fentanyl doses (placebo and cumulative 0.25, 0.75, and $1.5 \mathrm{mg} / 70 \mathrm{~kg}$ i.v.) and also functioned as an orientation session. Figure 1 (top row) shows the session 1 flowchart. After session baseline measures ( $30 \mathrm{~min}$ predrug), a nurse initiated four separate i.v. infusions at 20-min intervals $(t=0,20,40$, and $60 \mathrm{~min})$. Saline was administered at $t=0$, fentanyl $0.25 \mathrm{mg} / 70 \mathrm{~kg}$ at $t=20 \mathrm{~min}$, fentanyl $0.5 \mathrm{mg} / 70 \mathrm{~kg}$ at $t=40 \mathrm{~min}$, and fentanyl $0.75 \mathrm{mg} / 70 \mathrm{~kg}$ at $t=60 \mathrm{~min}$. This produced cumulative fentanyl doses of $0,0.25,0.75$, and
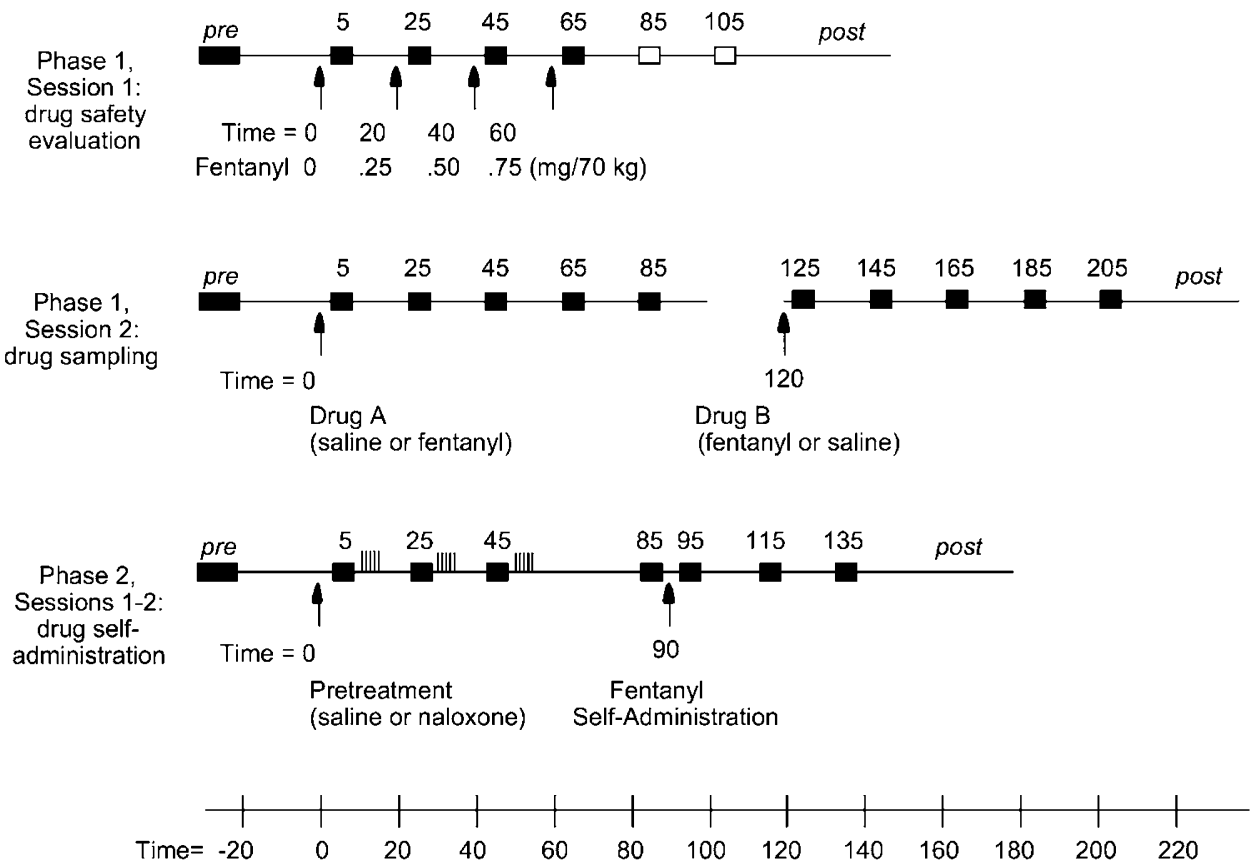

Figure I Flowchart for experimental sessions; see text for more details. Bottom: Timeline (min) for comparison across sessions. For all sessions, presession activities (pre) included urinalysis, intravenous (i.v.) catheter insertion, EEG scalp electrode placement, and baseline measures. Postsession activities (post) included vital signs, residual drug effects, sobriety test, and daily methadone dose. In all sessions, $t=0$ min refers to start of the first i.v. infusion. Arrows refer to infusions (time of infusion below each session timeline) and horizontal bars refer to EEG and pupil assessments following each infusion (time of assessment above each session timeline). Top row: In session I, four i.v. drug infusions were administered at 20-min intervals (saline at $t=0$ min, then ascending fentanyl doses at $t=20,40$, and 60 , which cumulated to $0.25,0.75$, and $1.5 \mathrm{mg} / 70 \mathrm{~kg}$, respectively). The EEG/pupil assessment period followed each infusion (shaded bars). Postdrug assessments (open bars; $t=85$ and $105 \mathrm{~min}$ ) measured recovery from drug effects, but these data were excluded from statistical analyses. Middle row: In session 2, two infusions, saline and fentanyl $1.5 \mathrm{mg} / 70 \mathrm{~kg}$, were administered $2 \mathrm{~h}$ apart ( $t=0$ and $120 \mathrm{~min}$ ). Postdrug assessments (shaded bars) were conducted at $t=5,25,45,65$, and 85 min after each infusion. Bottom row: In Phase 2, pretreatment (saline or naloxone) was administered at $t=0 \mathrm{~min}$, followed by assessments at $t=5,25$, and $45 \mathrm{~min}$. Five FR 100 opportunities (vertical lines) were presented at each of three times $(t=10,30$, and $50 \mathrm{~min})$. At $t=90 \mathrm{~min}$, the participant self-administered the response-contingent fentanyl dose. Assessments were conducted both before $(t=85 \mathrm{~min})$ and after fentanyl SA $(t=95,115$ and I $35 \mathrm{~min})$. 
$1.5 \mathrm{mg} / 70 \mathrm{~kg}$ at these four time points, respectively. From 3 to $6 \mathrm{~min}$ after each infusion dose, EEG data were collected during a 3-min rest period (ie supine, eyes-closed, and motionless in bed), followed by a pupil photo.

The purpose of session 2 was to allow participants to sample the fentanyl dose they could work for in later sessions, and to evaluate its effects relative to placebo. Figure 1 (middle row) presents the timeline for session 2. After baseline measures, participants sampled two separate i.v. drugs $2 \mathrm{~h}$ apart. These nurse-administered infusions were saline (placebo) and fentanyl $1.5 \mathrm{mg} / 70 \mathrm{~kg}$. For half the participants, the first infusion was placebo and for the others it was fentanyl (ie order was counterbalanced). From 3-6 min, 23-26 min, 43-46 min, 63-66 min, and 83-86 min after each infusion-referred to hereafter as 5, 25, 45, 65, and 85 min time points - the EEG and pupil evaluation was repeated.

Phase 2 evaluated the EEG and pupillary effects of fentanyl $(1.5 \mathrm{mg} / 70 \mathrm{~kg}$ bolus) SA relative to PA (phase 1), and whether naloxone relative to saline pretreatment (in two different sessions) attenuated EEG effects of fentanyl SA. After baseline measures, a research nurse initiated a pretreatment infusion $(t=0 \mathrm{~min})$, either naloxone $(0.2 \mathrm{mg} /$ $70 \mathrm{~kg}$ in the first participant and $0.1 \mathrm{mg} / 70 \mathrm{~kg}$ in all remaining participants) or saline. Immediately afterward, the participant was instructed he could work for drug in that day's session. (On other session days during Phase 2, which are irrelevant to the present study aims, each participant was given the opportunity to respond on the same schedule of reinforcement for an amount of money that he had rated in session 2 as being equivalent to fentanyl (see Greenwald, 2004, in press, for details). Moneymaintained responding was not statistically different than drug-maintained responding, and there were no significant EEG or pupil changes from baseline when participants responded to earn money (during anticipation of reinforcement) or at any time points after money was paid for operant performance.) After the pretreatment, the EEG and pupil evaluation was performed at $t=5,25,45$, and $85 \mathrm{~min}$ postdrug points.

At each of the times $(t=10,30$, and $50 \mathrm{~min})$ after each pretreatment, the participant could respond on five fixed ratio (FR) 100 schedules by pressing keys on the computer. Thus, there were 15 total opportunities to work for fentanyl in each SA session. Instructions were as follows: (1) the participant was told he could work for the total drug dose preferred in phase 1 (all participants preferred fentanyl $1.5 \mathrm{mg} / 70 \mathrm{~kg}$ over placebo in session 2 using a standard choice questionnaire); (2) each FR 100 completed earned 1/ 15 th $(0.1 \mathrm{mg} / 70 \mathrm{~kg})$ of the total available fentanyl dose; (3) on each FR 100 occasion, the participant could work for drug or not; (4) there was limited time to complete each FR 100 and that the clock would begin once the opportunity was signaled on the computer; (5) if the participant did not complete all 100 responses in $45 \mathrm{~s}$ then he did not earn $1 /$ 15 th of the drug; and (6) the participant was told that he would be able to initiate SA of the total drug dose he earned $90 \mathrm{~min}$ after the pretreatment. At $t=90 \mathrm{~min}$, the participant self-administered the fentanyl dose by pressing a button to start the infusion pump. The EEG and pupil evaluation was repeated at 5, 25, and $45 \mathrm{~min}$ post-fentanyl $(t=95,115$, and $135 \mathrm{~min}$ after the pretreatment). Therefore, the primary behavioral differences between PA (phase 1) and SA (phase 2) conditions were that participants (1) had to perform key press responses to earn drug, and (2) initiated infusion of the drug. Importantly, this schedule of reinforcement led to consistently high response rates (ca. 4.5/s) among these opioid-abusing volunteers (Greenwald, 2004, in press), who earned all 15 units of fentanyl under these conditions. Therefore, the dose of fentanyl during PA and SA phases was equated $(1.5 \mathrm{mg} / 70 \mathrm{~kg})$ in this study.

\section{Measures}

In each session, spontaneous EEG activity was recorded from gold-plated scalp electrodes applied with collodion using the International 10-20 system (Jasper, 1958). The 21lead EEG electrode montage included Fp1, Fp2, F3, F4, F7, F8, Fz, T3, T4, T5, T6, C3, C4, Cz, P3, P4, Pz, O1 and O2 placements, with each referenced to the contralateral earlobe. These monopolar EEG signals were collected with a Nicolet polygraph system (Ultrasom version SR 5.0) using low-frequency filter $=0.3 \mathrm{~Hz}$, high-frequency filter $=35 \mathrm{~Hz}$, and $60 \mathrm{~Hz}$ notch filter. Left and right electrooculogram (EOG) electrodes were applied to the outer canthi and referenced to contralateral earlobes to record horizontal eye movements. Electrode impedance was less than $5000 \mathrm{ohms}$ for each lead.

In all test phases and sessions, EEG activity was sampled at multiple 3-min intervals (separated in time), digitized at $512 \mathrm{~Hz}$ per lead, passed through an antialiasing filter, and subjected to Fast Fourier Transformation. For each 3-min interval, data were analyzed in seventy 2.56-s epochs for power spectra in the following frequency bands: delta (0.35$3.5 \mathrm{~Hz})$, theta $(3.5-7.5 \mathrm{~Hz})$, alpha $(7.5-11.5 \mathrm{~Hz})$, slow beta $(11.5-15.0 \mathrm{~Hz})$, and fast beta $(15-30 \mathrm{~Hz})$. Data from epochs with eye movements were excluded using an automated algorithm.

Initial examination of the data revealed that electrode interface pins at four frontal sites (Fp1, Fp2, F7 and F8) were faulty, and were therefore not analyzed. Data examination indicated no systematic laterality differences within conditions among most leads (ie F3/F4, C3/C4, P3/ P4, T5/T6 and O1/O2), except T3/T4 (ie over temporal cortex), and differences in EEG power within conditions sometimes occurred along the anterior/posterior midline axis $(\mathrm{Fz}-\mathrm{Cz}-\mathrm{Pz})$. To highlight representative results, data are reported for only five spatially distributed leads: three midline sites, along the anterior to posterior axis $(\mathrm{Fz}, \mathrm{Cz}$, $\mathrm{Pz}$ ) and left and right temporal sites (T3, T4) on either side of $\mathrm{Cz}$ (Figure 2, upper left). Since absolute EEG levels differed across individual participants and leads, data from each lead at each session time point were transformed to percentage change from predrug baseline scores, then averaged across participants to obtain an overall index of EEG response for statistical analyses.

After each EEG recording, pupil diameter was then assessed using a digital photography and scoring method (Greenwald et al, 1999). Pictures of the pupil were taken at a $10-\mathrm{cm}$ distance with a close-up lens and flash unit. All photographs were taken under dim, constant lighting conditions. Images were digitally filtered to enhance the contrast between pupil and iris, and scored for vertical and horizontal extent on a color monitor. The average of the 

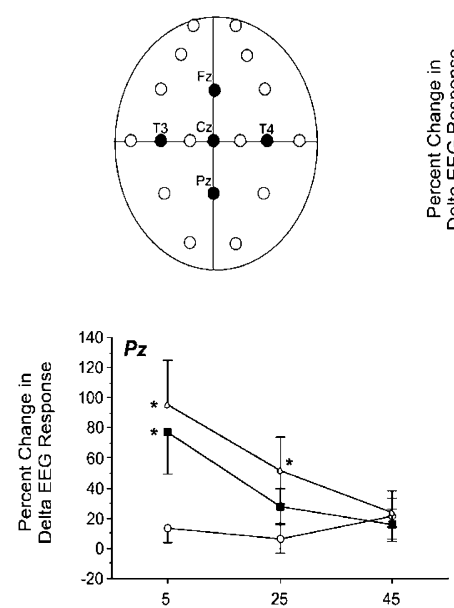
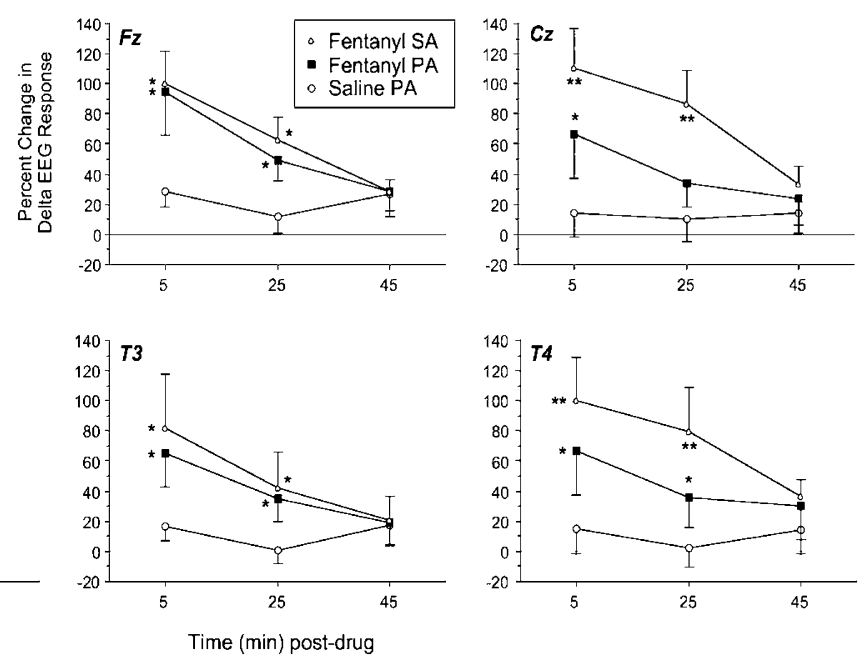

Figure 2 Delta-band EEG activation following self-administration (SA) and passive administration (PA) of fentanyl (I.5 mg/70 kg IV) and saline PA. Upper left: Scalp site location of five representative EEG leads (darkened circles) within the entire electrode array (open circles). Remaining images: Mean (+ I SEM) percent change in delta-band EEG response, relative to presession baseline levels, at these five leads: Fz (upper middle), Cz (upper right), Pz (lower left), T3 (lower middle), and T4 (lower right). Significantly greater response for fentanyl SA vs fentanyl PA was observed for leads Cz and T4, using leastsquare difference tests within a given time point. A single asterisk indicates that the condition mean (fentanyl SA and/or PA) was significantly greater than saline PA $(p<0.05)$, and a double asterisk indicates that the fentanyl SA mean was significantly greater than the fentanyl PA (as well as the saline) mean.

vertical and horizontal lengths, in pixel units, was used as the measure of pupil diameter.

\section{Data Analyses}

For Phase 1, session 1 data were subjected to Fentanyl Dose (placebo and cumulative $0.25,0.75$, and $1.50 \mathrm{mg} / 70 \mathrm{~kg}$ ) repeated measures analyses of variance (ANOVAs). In all analyses, Huynh-Feldt corrections were applied to adjust for violations of sphericity. ANOVAs with a significant $(p<0.05)$ dose effect were followed by least-square mean comparisons of active fentanyl dose conditions with placebo. To evaluate the significance of dose-related changes, the highest cumulative fentanyl dose condition was also compared with the two lower dose conditions. Phase 1, session 2 data were subjected to Drug (fentanyl, placebo $) \times$ Time $(5,25,45,65$, and 85 min postdrug change from baseline) repeated measures ANOVAs. Consistent with the expected time course of i.v. fentanyl effects from previous work (Greenwald et al, 1996), analyses revealed no carryover effects of administering fentanyl first (ie all measures returned to baseline levels).

Phase 2 data were analyzed using Pretreatment (naloxone and saline $) \times$ Time $(5,25$, and $45 \mathrm{~min}$ change from baseline) repeated measures ANOVAs. To separately determine effects of the pretreatments (assessment cycles starting at 5 , 25, and $45 \mathrm{~min}$, ie, prior to drug $\mathrm{SA}$ ) and reinforcer (assessment cycles starting at 5,25 , and $45 \mathrm{~min}$ post-SA), subsequent two-way ANOVAs were conducted using the relevant time points. To evaluate significant Pretreatment $\times$ Time interactions, experimental conditions were compared using least-square difference comparisons at the same postdrug times.

Response differences between self-administered and passively administered fentanyl bolus injections were evaluated using two-way repeated measures ANOVAs using three Conditions (fentanyl SA (phase 2), fentanyl PA, and saline PA (phase 1)) and three Time points $(5,25$, and
45 min postadministration). Least-square difference comparisons between conditions at each time point were conducted to further examine the source of these differences. (Although subjective drug effects were reported in the companion paper for this study (see Greenwald, 2004, in press), these measures were not tested for fentanyl SA vs PA differences as done here. To address this issue, five wellvalidated subjective drug effect measures (three visual analog scale ratings: pleasantness, drug liking, and drug high; a questionnaire measure of heroin craving based on a 34-item total score; and a questionnaire measure of opioid agonist symptoms based on a 16-item total score) were compared for fentanyl SA, fentanyl PA and saline PA conditions. Postdrug absolute peak scores were used in these analyses. For all five measures, ANOVA and least square difference comparison results were identical: Subjective response to fentanyl SA and fentanyl PA was not statistically different, and both were significantly different than saline PA. Fentanyl SA and PA increased pleasantness, liking, high and opioid agonist symptoms and decreased heroin craving, relative to saline. To summarize, there were no significant subjective effect differences between fentanyl SA and PA. These measures are not discussed further, as they were secondary to the aims of the present study.)

\section{RESULTS}

\section{PA (Phase 1)}

Table 1 presents results for PA fentanyl cumulative doseresponse in session 1. Relative to the placebo infusion (which produced no significant changes from baseline), fentanyl produced significant dose-related increases in EEG activity, with the highest dose increasing power in delta $(63-79 \%)$, theta $(76-128 \%)$, beta-1 $(31-60 \%)$, and beta-2 (49-92\%) frequency bands across the five selected leads, but there were no consistent alterations in alpha power (13-38\% across leads; data not shown). Fentanyl produced 
Table I Mean (SEM) Percent Change from Baseline in EEG Activation and Miosis from Passively Administered Cumulative Doses of Fentanyl (Phase I) $)^{\text {a }}$

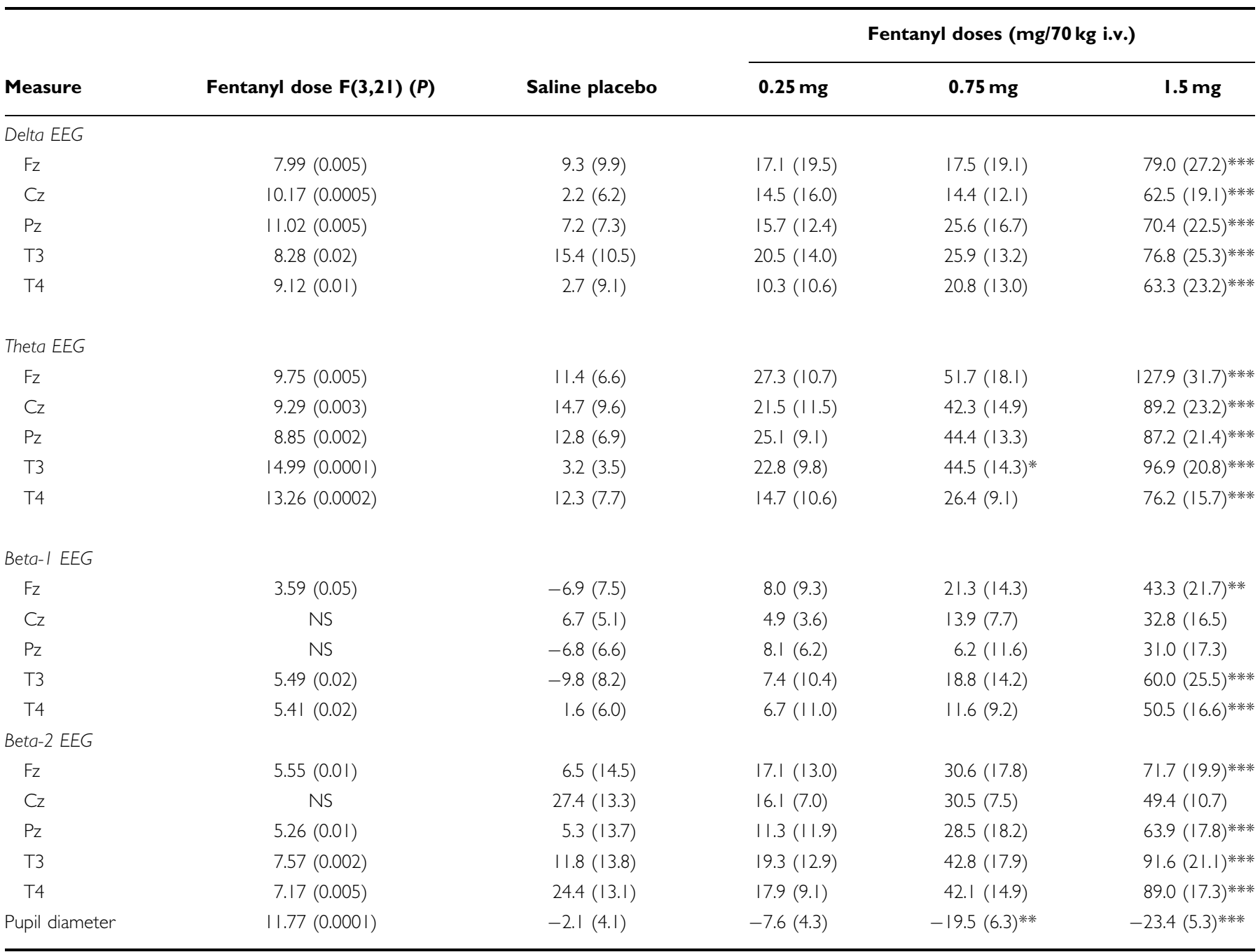

${ }^{a}$ Columns list the results of significance testing (ANOVAs) of the Fentanyl Dose effect (with Huynh-Feldt adjusted significance values, means \pm I SEM and comparisons at the four cumulative dose levels. Means are percent change from session baseline measurements (prior to saline placebo) taken 5 min after each infusion. Decreases in pupil diameter reflect constriction (miosis). EEG and pupil diameter scores before and after placebo did not significantly differ.

*Significantly greater than placebo. ** Significantly greater than fentanyl $0.25 \mathrm{mg}$ and placebo. **** Significantly greater than fentanyl $0.75 \mathrm{mg}, 0.25 \mathrm{mg}$, and placebo.

cumulative dose-related decreases in pupil diameter (miosis). Typically, only the highest cumulative fentanyl dose $(1.5 \mathrm{mg} / 70 \mathrm{~kg})$ produced EEG and miotic effects that significantly differed from placebo.

Table 2 summarizes results for bolus fentanyl vs placebo response in session 2. Relative to placebo (which again produced no significant changes from baseline), PA of highdose fentanyl yielded EEG effects that were not statistically different in magnitude and scalp distribution to those produced by the same cumulative fentanyl dose in session 1 . Figure 2 includes the time course of delta EEG activation for the fentanyl $1.5 \mathrm{mg} / 70 \mathrm{~kg}$ and saline placebo PA conditions. Relative to baseline, fentanyl significantly increased delta EEG amplitude at $5 \mathrm{~min}$ postdrug (average of $78 \%$ across leads; range, $65-94 \%$ ) and theta EEG amplitude (average of $66 \%$ across leads; range, $49-82 \%$ ); this fentanyl-placebo difference was smaller at $25 \mathrm{~min}$ postdrug, and was no longer evident at $45 \mathrm{~min}$ postdrug. Fentanyl also signifi- cantly increased beta- 1 and beta-2 EEG amplitude but only at two lead sites $(\mathrm{Cz}$ and $\mathrm{T} 4)$, whereas alpha amplitude (data not shown) was unaltered across scalp sites. Fentanyl bolus infusion significantly decreased pupil diameter by $24 \%$, a miotic effect that was similar in magnitude to session 1 .

\section{SA (Phase 2)}

Compared to session baseline, fentanyl SA (following pretreatment with saline) increased delta EEG amplitude an average of $104 \%$ across lead sites (Figure 2) and constricted pupil size by $19 \%$ at 5 -min postdrug. Fentanyl SA increased delta EEG amplitude significantly more than fentanyl PA (phase 1) at the central midline lead $(\mathrm{Cz})$ and right temporal lead (T4) at 5 and 25 min postdrug. Seven of the eight participants exhibited the predicted rank ordering of conditions (fentanyl SA $>$ fentanyl PA $>$ saline PA) for delta EEG at the $\mathrm{Cz}$ lead. There were no significant delta 
Table 2 Percent Changes from Baseline in Mean (SEM) 5-Min Post-Drug EEG Activation and Peak (SEM) Miosis Following Placebo and Fentanyl Passive Administration (PA; Phase I) and Fentanyl Self-Administration (SA; Phase 2) ${ }^{\text {a }}$

\begin{tabular}{|c|c|c|c|c|c|}
\hline Measure & Condition $F(2,14)(P)$ & Cond. $\times$ Time $F(4,28)(P)$ & Saline PA (Phase I) & Fentanyl PA (Phase I) & Fentanyl SA (Phase 2) \\
\hline $\mathrm{Fz}$ & $6.94(0.01)$ & $4.99(0.004)$ & $28.9(10.7)$ & $94.4(28.7)^{*}$ & $99.8(21.9)^{*}$ \\
\hline $\mathrm{Pz}$ & $3.59(0.06)$ & $5.52(0.002)$ & $13.5(9.7)$ & $76.8(27.6)^{*}$ & $95.3(9.6)^{*}$ \\
\hline T3 & NS & $4.23(0.02)$ & $16.4(9.3)$ & $65.1(21.8)^{*}$ & $82.0(35.6)^{*}$ \\
\hline \multicolumn{6}{|l|}{ Theta EEG } \\
\hline $\mathrm{Fz}$ & $5.23(0.04)$ & NS & $28.8(8.7)$ & $74.7(19.3)^{*}$ & $77.9(30.3)^{*}$ \\
\hline $\mathrm{Cz}$ & $5.78(0.03)$ & NS & $21.7(12.7)$ & $49.2(20.5)^{*}$ & $56.1(18.6)^{*}$ \\
\hline $\mathrm{Pz}$ & $3.67(0.09)$ & $2.92(0.05)$ & $26.8(13.7)$ & $49.5(16.0)^{*}$ & $51.4(21.3)^{*}$ \\
\hline T3 & $4.26(0.04)$ & $6.79(0.001)$ & $19.6(11.2)$ & $82.0(18.3)^{*}$ & $39.4(21.1)^{* * *}$ \\
\hline \multicolumn{6}{|l|}{ Beta- / EEG } \\
\hline $\mathrm{Fz}$ & NS & NS & $23.9(9.9)$ & $31.4(11.7)$ & $47.0(20.4)$ \\
\hline $\mathrm{Cz}$ & $3.05(0.08)$ & NS & $0.9(5.8)$ & $19.8(8.6)$ & $29.7(\mid 1.7)$ \\
\hline $\mathrm{Pz}$ & NS & NS & $22.6(13.4)$ & $17.2(9.7)$ & $24.5(11.6)$ \\
\hline T3 & NS & NS & $29.8(12.7)$ & $43.6(20.1)$ & $41.2(21.7)$ \\
\hline $\mathrm{T} 4$ & $3.13(0.08)$ & NS & | 4.5 (9.9) & $37.0(14.6)$ & $61.2(10.8)$ \\
\hline \multicolumn{6}{|l|}{ Beta-2 EEG } \\
\hline $\mathrm{Fz}$ & NS & NS & $33.8(14.2)$ & $46.6(10.3)$ & $59.5(18.6)$ \\
\hline $\mathrm{Cz}$ & $3.92(0.05)$ & NS & $2.4(6.5)$ & $36.8(12.9)^{*}$ & $52.1(14.3)^{*}$ \\
\hline $\mathrm{Pz}$ & NS & NS & $31.3(13.0)$ & $43.8(12.5)$ & $63.2(22.1)$ \\
\hline
\end{tabular}

${ }^{a}$ Columns list the results of significance testing (ANOVAs) of the Condition main effect and Condition $\times$ Time interaction for all measures (with Huynh-Feldt adjusted significance values), means + I SEM, and comparisons. Means are percent change from session baseline. EEG response is the increase at 5-min post-drug. Parallel analysis of post-drug peak (data not shown) yielded similar statistical results because peak scores usually occurred at 5 min post-drug. Pupil response is the smallest value (miosis) from time points at 5, 25, or 45 min post-drug; there was no Condition $\times$ Time interaction tested for pupil diameter.

*Significantly greater than saline PA. **Significantly different than fentanyl PA.

EEG amplitude differences between fentanyl SA and PA for the remaining leads (Table 2). Fentanyl SA tended to decrease theta EEG amplitude relative to fentanyl PA (Table 2) at the left and right temporal leads (T3 and T4), but this was only significant at T3. Fentanyl SA and PA produced similar increases in EEG response for other EEG frequency bands (alpha, beta-1, and beta-2) and similar degree of pupil constriction (Table 2).

As shown in Figure 3, naloxone itself produced no significant effect on EEG activity (time points before $t=90 \mathrm{~min}$ ), but it did significantly attenuate fentanyl SAinduced EEG activity (time points after $t=90 \mathrm{~min}$ ). Naloxone pretreatment increased pupil diameter $12 \%$ relative to session baseline (ie mydriasis associated with precipitated opioid withdrawal), then returned to baseline $85 \mathrm{~min}$ after pretreatment; naloxone did not significantly attenuate fentanyl SA-induced pupil constriction.

\section{DISCUSSION}

The seminal finding of this preliminary clinical study is that self-administered fentanyl doubled delta EEG power, whereas the identical dose of passively administered fentanyl produced a statistically smaller increase in delta EEG power, in heroin abusers. These initial findings are consistent with preclinical data that self- and passively administered opioids produce different patterns of electrophysiological activity (Chang et al, 1997, 1998; Grasing and Szeto, 1993; Kiyatkin and Rebec, 2001; Lee et al, 1999) and various other neurobiological changes (Jacobs et al, 2003). Fentanyl, a highly potent, short-acting, heroin-like drug with abuse potential in humans (Greenwald et al, 1996; Baylon et al, 2000), functioned as a reinforcer (ie it maintained high rates of responding) in these opioiddependent volunteers. In the larger-scale study during 


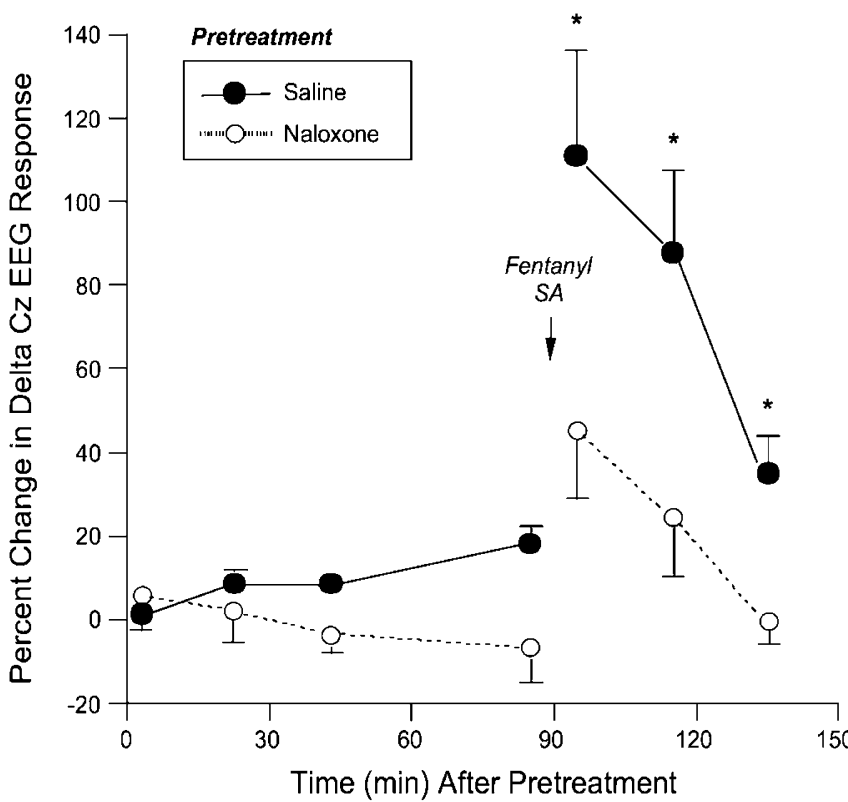

Figure 3 Delta-band EEG activity at the central midline $(C z)$ lead, following saline (control) and naloxone pretreatment. Naloxone relative to saline pretreatment did not significantly alter EEG response through $85 \mathrm{~min}$ after pretreatment, but naloxone attenuated the fentanyl SA-induced deltaband EEG increase, suggesting the involvement of opioid receptors. Asterisks indicate that EEG response was significantly higher $(p<0.05$, leastsquare difference tests) at all three time points after fentanyl selfadministration with saline, relative to naloxone, pretreatment.

which these EEG data were recorded, these fentanyl doses significantly increased self-reported euphoria, decreased self-reported heroin craving, and significantly increased ratings of the drug's monetary value (Greenwald, 2004, in press). Interestingly, SA and PA fentanyl did not produce significantly different effects nor any trends on subjective drug liking, high, opioid agonist symptoms, pleasantness, or heroin craving, in the present analysis (see section Data Analysis), indicating a dissociation between EEG and subjective measures. The reason for this dissociation between EEG and subjective report measures is not presently clear but could reflect factors other than direct pharmacological effects of fentanyl on the EEG (see below).

Although the present data await replication, the specificity of this novel finding was demonstrated in several ways. First, although fentanyl PA increased EEG amplitude in delta, theta, beta-1, and beta-2 (ie slow- and fast-wave) frequency bands, the greater EEG response following fentanyl SA was delta-specific $(0.35-3.5 \mathrm{~Hz})$. Interestingly, this is the frequency range in which fentanyl produces its typical effects when administered passively to surgical patients (Bovill et al, 1983; Scott et al, 1991; Sebel et al, 1981; Wauquier et al, 1984). Unfortunately, there are no published studies that compare postoperative EEG response to self-administered opioids (ie patient-controlled analgesia) $v s$ medical staff-administered opioids. Second, the delta power increase was primarily evident at the central midline lead $(\mathrm{Cz})$ and the right temporal (T4) lead. At the same time, fentanyl SA relative to PA had the opposite effect on theta-band power, that is, significantly decreased activation at the left temporal (T3) lead. As scalp EEG signal changes are complexly determined and must be cautiously inter- preted, the meaning of these sites of SA-related activity is not presently clear. Third, EEG activity changes produced by fentanyl PA and SA were time-dependent, peaking at the first postdrug time point and decreasing thereafter. These observed changes in EEG signal are consistent with fentanyl's pharmacokinetics. Fourth, naloxone (relative to saline) pretreatment attenuated fentanyl SA-induced EEG activity, also in a time-related manner. This finding is most likely related to lingering opioid receptor antagonist actions of naloxone. Finally, although pupil constriction - a classical physiological measure of $m u$-opioid agonist effect - was sensitive to fentanyl dose, it was not sensitive to the difference in fentanyl SA vs PA.

There are several limitations to the present study. First, the sample size was small and it will be necessary to conduct a larger-scale study to test the reliability of these observed effects. Second, experimental manipulations were conducted within subject (ie each participant was his own control), in contrast to the between-subject triad, yokedcontrol design that has been traditionally used in preclinical studies. Ideally, the most convincing human demonstration would be to conduct a triad (parallel-group) design that mimics these preclinical studies. However, a potential drawback with such an approach is that there are substantial individual differences across human subjects, which could obscure drug SA vs PA differences using a yoked-control design. Thus, despite these limitations, a within-subject design may be more sensitive and practical than a between-subjects design to address this scientific question with human volunteers. The primary behavioral differences between SA and PA conditions in this study were that each participant had to perform 1500 key press responses to earn drug, and they initiated drug infusion with a single button press. It is possible that differences in EEG response to SA vs PA could partly depend on the operant response requirement, such that a lower response requirement than imposed here would fail to produce this neurophysiological effect; therefore, it would be useful to study this factor parametrically. On the other hand, the temporally extended operant response criterion in this study raises the possibility that the EEG difference between SA and PA may be influenced by factors other than sheer motor output. For instance, the intermittent work periods (ie three periods of five FR 100 performances, each separated by $20 \mathrm{~min}$ ) and delay between completing the work requirement and self-administering drug (ie about $30 \mathrm{~min}$ ) in the present study could introduce effects of associative cognitive processes (eg anticipation of initiating the infusion and the euphoria it produces) that may affect EEG response. Additional research should also address these different influences.

A third limitation is that fentanyl PA always occurred before fentanyl SA in the present study, which confounds the effects of these different modes of drug administration with time. Typically, one observes a reduction in effect (ie tolerance) with repeated fentanyl administration; tolerance has been reported for fentanyl's discriminative stimulus effects (Emmett-Oglesby et al, 1989; Walker et al, 1997) as well as antinociceptive and sedative effects (Choe and Smith, 2000; Chia et al, 1999; Thornton et al, 2000). Although there do not appear to be any published studies that have examined whether repeated fentanyl exposure 
produces sensitization, studies have shown that repeated morphine exposure produces tolerance to EEG effects (Tortella et al, 1979; Young and Khazan, 1984) but sensitization to locomotor behavior (Bartoletti et al, 1987; Powell and Holtzman, 2001). Thus, preclinical data suggest that one would expect repeated fentanyl exposure to result in decreased EEG response, in contrast to the increased response following SA observed in later sessions (relative to PA in earlier sessions) of the present study. However, it is not clear whether such data are comparable to the human experimental situation for a few reasons. First, the present study examined effects of repeated fentanyl exposure in heroin-dependent humans, whereas preclinical studies have examined effects of repeated opioid exposure in previously opioid-naïve animals. Second, there are major differences in dosing procedures between human and animal studies that make close comparisons difficult. A related issue is that the present conclusions may be limited to the fentanyl dose used in this study; additional studies should vary the drug unit dose to explore the robustness of these findings. Third, different opioids such as morphine and fentanyl can produce markedly different EEG patterns. For instance, Phillips et al (1994) found that morphine primarily affected alpha-band but not delta-band activity in heroin abusers, whereas in the present study fentanyl primarily affected delta-band but not alpha-band activity.

The present authors are not aware of any published clinical studies that have sought to examine the effect of drug SA $v s$ PA on any measure of human brain function. In laboratory rats, total EEG amplitude (a gross functional measure that is not frequency-specific) was observed to be lower preceding morphine SA than preceding PA, which the authors (Grasing and Szeto, 1993) suggested was probably related to the lever-pressing behavior of the group of animals who could self-administer morphine. Unfortunately, results of that study did not include whether the post-SA $v s$ post-PA EEG response to morphine differed, making it difficult to compare with the present results. In other animal studies, freely moving rats that actively self-administered (FR 1 schedule) vs passively received intravenous heroin showed different patterns of electrophysiological activity in both the ventral tegmental area (Kiyatkin and Rebec, 2001) and nucleus accumbens and prefrontal cortex (Chang et al, 1997, 1998; Lee et al, 1999). Data from these latter studies are consistent in showing that, although most neurons showed no changes in rate of spontaneous firing after heroin SA or PA and very few neurons $(<5 \%)$ showed any post-heroin excitatory response, about $20 \%$ of nucleus accumbens neurons selectively responded with inhibition of spontaneous firing following heroin SA relative to PA, an effect that was blocked by systemic naloxone pretreatment (Lee et al, 1999). Of course, the interpretation of the present study results is much more limited because scalp EEG activity cannot precisely measure localized brain function; that is, it lacks the specificity of single-unit firing used in these preclinical studies to identify neurobiological processes involved in drug reinforcement.

Tempered by the limitations described, it appears that the significantly greater EEG activation produced by fentanyl SA relative to PA in this study may favor the involvement of a tolerance-opposing process, which could include altera- tions in attention, cognitive associative processes, and/or behavioral activity preceding and contingent upon drug self-administration. The potential contributions of these multiple factors should be tested to address these alternative explanations. With these cautions in mind, the positive results of this 'proof of concept' test support the idea that real-time neuroimaging (eg functional MRI) techniques could be used to study more precisely the interaction between brain circuits that mediate drug SA behaviors and direct pharmacological effects. This type of research could provide insights into learning processes that make drug addiction such a persistent psychobiological disorder and challenging public health problem.

\section{ACKNOWLEDGEMENTS}

This research was supported by NIH/NIDA R29 DA11079 and Joseph Young, Sr. funds from the State of Michigan to Mark Greenwald. We thank Ken Bates for recruiting volunteers; Ja'Near Mathis for urine toxicology testing; Drs John Hopper and Karen Saules for diagnostic screening; Dawanda Cooper for preparing methadone, and Linda McIntyre for preparing experimental drugs. For test sessions, Suzanne Manji coordinated the research team; Rita Hill, Irish Leonardo, and Carol Seamon provided nursing assistance; Drs Debra Glitz and Manuel Tancer provided medical coverage; and Doug Ditri, Christopher LaRose, and Julie Scallen collected all data.

\section{REFERENCES}

Amit Z, Brown ZW, Sklar LS (1976). Intraventricular selfadministration of morphine in naive laboratory rats. Psychopharmacology (Berl) 48: 291-294.

Bartoletti M, Gaiardi M, Gubellini C, Bacchi A, Babbini M (1987). Previous treatment with morphine and sensitization to the excitatory actions of opiates: dose-effect relationship. Neuropharmacology 26: 115-119.

Baylon GJ, Kaplan HL, Somer G, Busto UE, Sellers EM (2000). Comparative abuse liability of intravenously administered remifentanil and fentanyl. J Clin Psychopharmacol 20: 597-606.

Bovill JG, Sebel PS, Wauquier A, Rog P, Schuyt HC (1983). Influence of high-dose alfentanil anaesthesia on the electroencephalogram: correlation with plasma concentrations. $\mathrm{Br} J$ Anaesth 55(Suppl 2): 199-209.

Chang JY, Janak PH, Woodward DJ (1998). Comparison of mesocorticolimbic neuronal responses during cocaine and heroin self-administration in freely moving rats. J Neurosci 18: 3098-3115.

Chang JY, Zhang L, Janak PH, Woodward DJ (1997). Neuronal responses in prefrontal cortex and nucleus accumbens during heroin self-administration in freely moving rats. Brain Res 754: 12-20.

Chia YY, Liu K, Wang JJ, Kuo MC, Ho ST (1999). Intraoperative high dose fentanyl induces postoperative fentanyl tolerance. Can J Anaesth 46: 872-877.

Choe CH, Smith FL (2000). Sedative tolerance accompanies tolerance to the analgesic effects of fentanyl in infant rats. Pediatr Res 47: 727-735.

Dworkin SI, Mirkis S, Smith JE (1995). Response-dependent versus response-independent presentation of cocaine: differences in the lethal effects of the drug. Psychopharmacology (Berl) 117: 262-266.

Emmett-Oglesby MW, Shippenberg TS, Herz A (1989). Fentanyl and morphine discrimination in rats continuously infused with fentanyl. Behav Pharmacol 1: 3-11. 
Fink M, Zaks A, Volavka J, Roubicek J (1971). Electrophysiological studies of opiates and antagonists in man. In: Clouet DH (ed). Narcotic Drugs, Biochemical Pharmacology. Plenum: New York, 452-467.

First MB, Spitzer RL, Gibbon M, Williams JBW (1996). Structured Clinical Interview for DSM-IV Axis Disorders-Patient Edition (SCID-I/P, Version 2.0). Biometrics Research Department, NY State Psychiatric Institute: New York.

Grasing K, Szeto H (1993). EEG changes with different levels of morphine self-administration. Neuropharmacology 32: 543-553.

Greenwald MK (2002). Heroin craving and drug use in opioidmaintained volunteers: effects of methadone dose variations. Exp Clin Psychopharmacol 10: 39-46.

Greenwald MK (2004). Opioid craving and seeking behavior in physically dependent volunteers: effects of acute withdrawal and drug reinforcement opportunity. Exp Clin Psychopharmacol (in press).

Greenwald MK, DiCerbo L, Kish D, Kuennen J (1999). Description and reliability of a new method for digital measurement of pupil diameter. NIDA Res Monogr. National Institute on Drug Abuse: Rockville, Maryland, 179: 311.

Greenwald MK, June HL, Stitzer ML, Marco AP (1996). Comparative clinical pharmacology of short acting mu opioids in drug abusers. J Pharmacol Exp Ther 277: 1228-1236.

Hemby SE, Martin TJ, Co C, Dworkin SI, Smith JE (1995). The effects of intravenous heroin administration on extracellular nucleus accumbens dopamine concentrations as determined by in vivo microdialysis. J Pharmacol Exp Ther 273: 591-598.

Jacobs EH, de Vries TJ, Smit AB, Schoffelmeer AN (2004). Gene transcripts selectively down-regulated in the shell of the nucleus accumbens long after heroin self-administration are up-regulated in the core independent of response contingency. FASEB J 18: 200-202.

Jacobs EH, Smit AB, de Vries TJ, Schoffelmeer AN (2003). Neuroadaptive effects of active versus passive drug administration in addiction research. Trends Pharmaceutical Sci 24: 566-573.

Jacobs EH, Spijker S, Verhoog CW, Kamprath K, de Vries TJ, Smit $\mathrm{AB}$ et al (2002). Active heroin administration induces specific genomic responses in the nucleus accumbens shell. FASEB J 16: 1961-1963.

Jasper HH (1958). The ten-twenty electrode system of the international federation. EEG Clin Neurophysiol 10: 371-375.

Kiyatkin EA, Rebec GV (2001). Impulse activity of ventral tegmental area neurons during heroin self-administration in rats. Neuroscience 102: 565-580.

Lee RS, Criado JR, Koob GF, Henriksen SJ (1999). Cellular responses of nucleus accumbens neurons to opiate-seeking behavior: I. Sustained responding during heroin self-administration. Synapse 33: 49-58.

National Institute on Drug Abuse Community Epidemiology Work Group (2002). Epidemiological Trends in Drug Abuse. National Institute on Drug Abuse: Rockville, Maryland.

Phillips RL, Herning R, London ED (1994). Morphine effects on the spontaneous electroencephalogram in polydrug abusers: corre- lations with subjective self-reports. Neuropsychopharmacology 10: $171-181$

Powell KR, Holtzman SG (2001). Parametric evaluation of the development of sensitization to the effects of morphine on locomotor activity. Drug Alcohol Depend 62: 83-90.

Scott JC, Cooke JE, Stanski DR (1991). Electroencephalographic quantitation of opioid effect: comparative pharmacokinetics of fentanyl and sufentanil. Anesthesiology 74: 34-42.

Sebel JS, Bovill JG, Wauquier A, Rog P (1981). Effects of high-dose fentanyl anesthesia on the electroencephalogram. Anesthesiology 55: 203-211.

Self DW, McClenahan AW, Beitner-Johnson D, Terwilliger RZ, Nestler EJ (1995). Biochemical adaptations in the mesolimbic dopamine system in response to heroin self-administration. Synapse 21: 312-318.

Shipley B (1967). The Shipley Institute of Living Scale. Western Psychological Services: Los Angeles, California.

Smith JE, Co C, Freeman ME, Lane JD (1982). Brain neurotransmitter turnover correlated with morphine-seeking behavior of rats. Pharmacol Biochem Behav 16: 509-519.

Smith JE, Co C, Freeman ME, Sands MP, Lane JD (1980). Neurotransmitter turnover in rat striatum is correlated with morphine self-administration. Nature 287: 152-154.

Smith JE, Co C, Lane JD (1984a). Limbic acetylcholine turnover rates correlated with rat morphine-seeking behaviors. Pharmacol Biochem Behav 20: 429-442.

Smith JE, Co C, Lane JD (1984b). Limbic muscarinic cholinergic and benzodiazepine receptor changes with chronic intravenous morphine and self-administration. Pharmacol Biochem Behav 20: $443-450$.

Thornton SR, Lohmann AB, Nicholson RA, Smith FL (2000). Fentanyl self-administration in juvenile rats that were tolerant and dependent to fentanyl as infants. Pharmacol Biochem Behav 65: $563-570$.

Tortella FC, Moreton JE, Khazan N (1979). Electroencephalographic and behavioral tolerance to and cross-tolerance between D-Ala2-methionine-enkephalinamide and morphine in the rat. J Pharmacol Exp Ther 210: 174-179.

Volavka J, Zaks A, Roubicek J, Fink M (1970). Electrographic effects of diacetylmorphine (heroin) and naloxone in man. Neuropharmacology 9: 587-593.

Walker EA, Richardson TM, Young AM (1997). Tolerance and cross-tolerance to morphine-like stimulus effects of mu opioids in rats. Psychopharmacology (Berl) 133: 17-28.

Wauquier A, Bovill JG, Sebel PS (1984). Electroencephalographic effects of fentanyl-, sufentanil- and alfentanil anesthesia in man. Neuropsychobiology 11: 203-206.

Welzl H, Kuhn G, Huston JP (1989). Self-administration of small amounts of morphine through glass micropipettes into the ventral tegmental area of the rat. Neuropharmacology 28: 1017-1023.

Young GA, Khazan N (1984). Differential tolerance and crosstolerance to repeated daily injections of mu and kappa opioid agonists in the rat. Neuropharmacology 23: 505-509. 